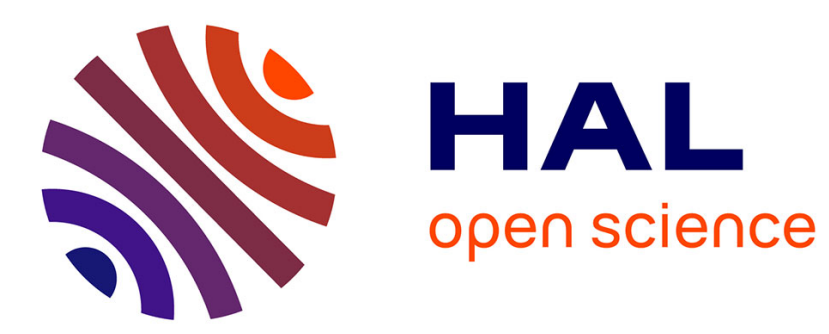

\title{
Rough thin pavement thickness estimation by GPR
}

Nicolas Pinel, Cédric Le Bastard, Limei Liu, Christophe Bourlier, Yide Wang

\section{To cite this version:}

Nicolas Pinel, Cédric Le Bastard, Limei Liu, Christophe Bourlier, Yide Wang. Rough thin pavement thickness estimation by GPR. International Geoscience and Remote Sensing Symposium, Jul 2009, Le Cap (Cape Town), South Africa. pp.1255. hal-00426435

\section{HAL Id: hal-00426435 https://hal.science/hal-00426435}

Submitted on 26 Oct 2009

HAL is a multi-disciplinary open access archive for the deposit and dissemination of scientific research documents, whether they are published or not. The documents may come from teaching and research institutions in France or abroad, or from public or private research centers.
L'archive ouverte pluridisciplinaire HAL, est destinée au dépôt et à la diffusion de documents scientifiques de niveau recherche, publiés ou non, émanant des établissements d'enseignement et de recherche français ou étrangers, des laboratoires publics ou privés. 


\section{ROUGH THIN PAVEMENT THICKNESS ESTIMATION BY GPR}

\author{
N. Pinel, L. Liu, C. Bourlier, Y. Wang \\ IREENA Laboratory, Université de Nantes \\ Polytech'Nantes \\ Nantes, France
}

\author{
C. Le Bastard \\ LRPCA \\ ERA 17 \\ Les Ponts de Cé, France
}

\begin{abstract}
In civil engineering, usually the methods used to estimate the thickness of thin pavements consider flat interfaces for simplification. In this paper, the roughness of the surfaces is taken into account. First, the amplitudes of the first two echoes from the rough thin pavement are calculated from a rigorous electromagnetic method, the PILE method. A comparison is then made with the flat interface case, and their differences in the electromagnetic backscattering are highlighted. Eventually, the influence of the pavement roughness on the pavement thickness estimation is investigated by using the Maximum Likelihood Method.
\end{abstract}

Index Terms - Radar scattering, Electromagnetic scattering by rough surfaces, Ground wave propagation, Nondestructive testing, Delay estimation

\section{INTRODUCTION}

Ground penetrating radar (GPR) is a useful means of media sounding, which is widely used at centimeter-scale wavelengths in road surfaces evaluation [1, 2, 3]. In this context, the roadway is usually considered as made up of perfectly flat stratified interfaces. Then, the vertical structure of the roadway is deduced from radar echo detection and amplitudes estimation. Echo detection provides the time-delay estimation (TDE) associated with each interface, and amplitude estimation is used to retrieve the wave speed within each layer. The case of small pavement thicknesses was studied in a recent paper [3].

In general, classical methods of pavement thickness estimation assume flat interfaces for the pavement. Even if this first approximation has sense and is rather realistic, to our knowledge the validity domain of this approximation, and its influence on the electromagnetic backscattering and on the GPR process have not been studied in details. This is the scope of this work. Thus, in this paper, the surface roughness of the pavement is taken into account in the electromagnetic backscattering modeling and in the GPR thickness estimation process, and compared with the case of neglecting the roughness of the pavement.
First, the amplitudes of the first two echoes from the rough thin pavement are calculated with a rigorous electromagnetic method, namely the PILE method [4]. The frequency behavior of the echoes is then presented in the considered frequency band, $f \in[1.0 ; 3.0] \mathrm{GHz}$, comparatively to the echoes obtained for flat interfaces. Finally, the influence of the pavement roughness on the thickness estimation is investigated by using the Maximum Likelihood Method.

\section{ECHO AMPLITUDES: FREQUENCY BEHAVIOR}

In this section, the frequency behavior of the first two backscattered echoes $s_{1}$ and $s_{2}$ of a rough pavement is presented. To calculate the echoes within the frequency band $f \in[1.0 ; 3.0] \mathrm{GHz}$, the PILE (Propagation Inside Layer Expansion) method [4] is used. It is a Method-of-Moments based method which is able to compute rigorously each echo reflected by a flat or a rough layer.

\subsection{Simulation parameters}

The pavement under study is an Ultra Thin Asphalt Surfacing (UTAS) of thickness $H=20 \mathrm{~mm}$ [5], overlying a rolling band of same general composition. It is assumed that the UTAS and the rolling band can be assimilated to homogeneous media at the frequency band under study $f \in[1.0 ; 3.0]$ $\mathrm{GHz}[3,6,7]$. Their relative permittivities typically range between 4 and 8 [8], and their conductivities between $10^{-3}$ and $10^{-2} \mathrm{~S} / \mathrm{m}$ [9]. For the simulations, their relative permittivities are taken as $\epsilon_{r 2}=5$ and $\epsilon_{r 3}=8$, respectively, and their conductivities as $\sigma_{2}=5 \times 10^{-3} \mathrm{~S} / \mathrm{m}$ and $\sigma_{3}=10^{-2} \mathrm{~S} / \mathrm{m}$, respectively. The two rough interfaces $\Sigma_{A}$ and $\Sigma_{B}$ are assumed to be described by a Gaussian height probability density function (pdf), and an exponential auto-correlation function [10, 11]. For the upper interface $\Sigma_{A}$, the root mean square (rms) height $\sigma_{h A}$ is of the order of $1 \mathrm{~mm}$, and the correlation length $L_{c A}$ of the order of $5-10 \mathrm{~mm}$. For the lower interface $\Sigma_{B}$, the rms height $\sigma_{h B}$ and the correlation length $L_{c B}$ are a bit greater. For the simulations, the chosen parameters are $\sigma_{h A}=0.8 \mathrm{~mm}, L_{c A}=10.0 \mathrm{~mm}, \sigma_{h B}=1.6 \mathrm{~mm}$, and $L_{c B}=30.0 \mathrm{~mm}$. In practice, the two rough surfaces are 
only very weakly correlated, so that it can be assumed here that they are statistically uncorrelated.

Concerning the incident wave, a monochromatic incident wave of TM polarization (also called vertical polarization) is considered, with normal incidence onto the pavement, $\theta_{i}=0$. The emitter antenna is assumed to be in the far-field zone of the ground, so that the incident wave is assumed to be plane. The typical width of the central zone illuminated by the emitter antenna is of the order of $100-200 \mathrm{~mm}$ [12]. Then, for the simulations of the numerical method, surfaces of length $L=600 \mathrm{~mm}$ will be considered, illuminated by a Thorsos beam of attenuation parameter $g=L / 6$. A normal incident wave (with incidence angle $\theta_{i}=0$ ) is taken, and the first two orders of the reflected echoes by the rough layer $s_{1}$ and $s_{2}$ are calculated under the PILE method. Then, to determine the frequency behavior of the received echoes $s_{k} \equiv s_{k}(f)$ (see equation (2) of [3]) in the backscattering direction $\theta_{s}=\theta_{i}=0$, it is necessary to run the numerical simulation scenario at different frequencies $f$ within the considered bandwidth, $f \in[1.0 ; 3.0] \mathrm{GHz}$.

The numerical process is described as follows. The rough layer, with two independent rough surfaces, is generated by a Monte-Carlo process (the two rough surfaces being generated from independent processes), for which the calculation of the backscattered signals $s_{1}$ and $s_{2}$ is led with the PILE. In order to study the variability of the echo amplitudes $s_{1}$ and $s_{2}$, several independent Monte-Carlo processes are generated. Thus, it is possible to estimate the standard deviations of the echo amplitudes, and even a profile of their calculated probability density functions if a significant number of realizations is led (typically, of the order of 10000 [13]). Indeed, for a practical scenario, the illuminated surface area is of the order of $100-200 \mathrm{~mm}$, which is not large in comparison with the two surface correlation lengths $l_{c A}=10.0 \mathrm{~mm}$ and $l_{c B}=30.0$ $\mathrm{mm}$. This implies that the received echo amplitudes depend on the location of the pavement where the measurement is made. As a consequence, in order to study the variability of the received echo amplitudes, a significant number of realizations must be generated.

To compute the numerical results, at least 1000 independent realizations of a Monte-Carlo process are generated, in order to simulate the variability of the received echoes. For the simulation of the numerical method, the two rough interfaces are sampled with a sampling step $\Delta x=\lambda_{2} / 10$, with $\lambda_{2}$ the wavelength inside the inner medium $\Omega_{2}$.

\subsection{Numerical results}

First, numerical simulations are led at a fixed radar frequency $f$, in the middle of the radar band under study, i.e. $f=2.0$ $\mathrm{GHz}$. In order to study the probability density function (pdf) of the echoes $s_{1}$ and $s_{2}$, the number of realizations of the Monte-Carlo process is taken as 10000 . A comparison is also made between the case with rough interfaces and the case
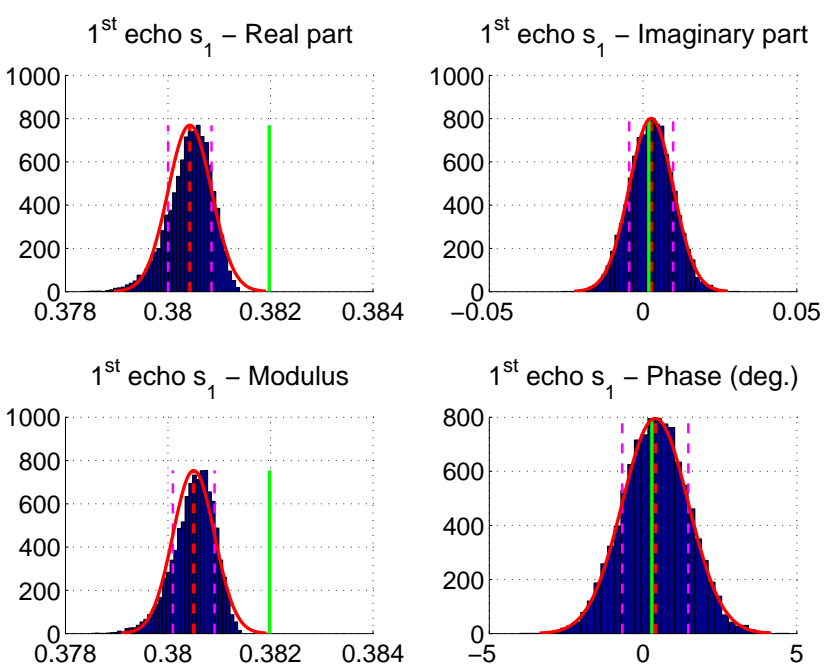

Fig. 1. Probability density functions (pdf) of the first echo $s_{1}$ (real part, imaginary part, modulus and phase) obtained from 10000 realizations, at a radar frequency $f=2 \mathrm{GHz}$. The mean value is plotted in red dashed vertical line, and the mean value plus and minus the standard deviation are plotted in purple dashed vertical line. Then, the pdf is compared with a Gaussian pdf having the same mean value and standard deviation. Comparison is also made with the flat case in green vertical line.
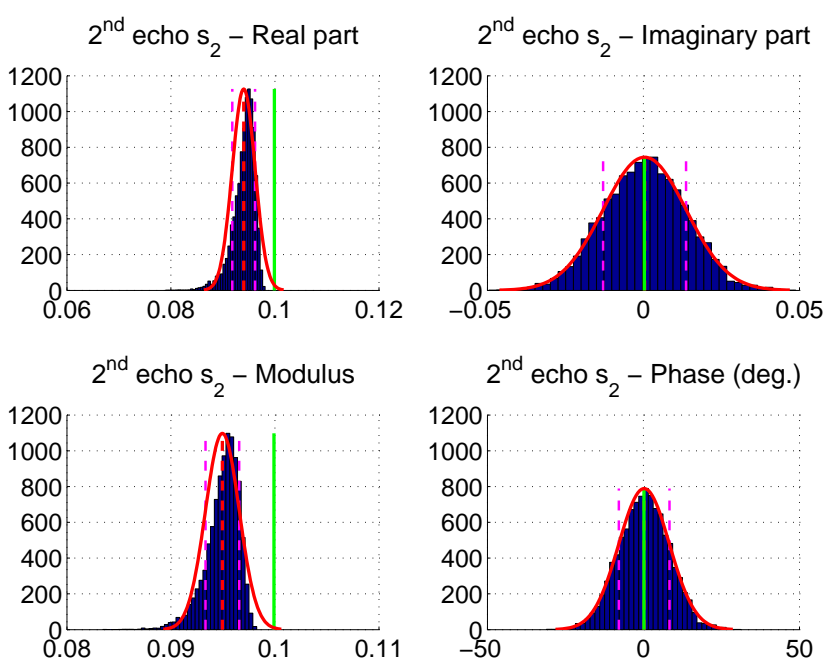

Fig. 2. Same simulation parameters as in Fig. 1, but for the second echo $s_{2}$

with flat interfaces. Numerical results of the simulated pdfs are plotted in Fig. 1 for the first echo $s_{1}$, and in Fig. 2 for the second echo $s_{2}$. In each figure, the pdf of the real part, the imaginary part, the modulus, and the phase (in degrees) of the echo are represented. The mean value is plotted in red dashed vertical line, and the mean value plus and minus the standard deviation are plotted in purple dashed vertical line. Then, the 


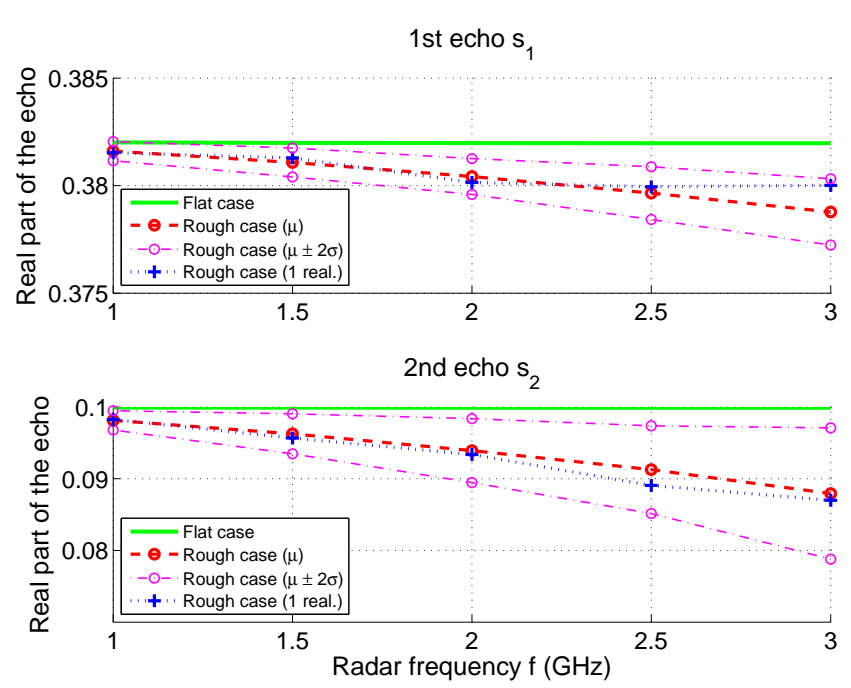

Fig. 3. Frequency behavior of the real part of the first two echoes $s_{1}$ and $s_{2}$

pdf is compared with a Gaussian pdf having the same mean value and standard deviation in full red line. A comparison is also made with the case of flat interfaces (whose pdf is a Dirac delta function) in green vertical line.

Concerning the first echo $s_{1}$, the imaginary part and the phase do not highlight a significant difference between the flat case and the mean value of the rough case. Moreover, the dispersion around this mean value remains low: for instance, in the phase distribution, the RMS phase is of the order of 1 degree. By contrast, a relatively small but though significant difference occurs in the real part and in the modulus. As expected, the (upper) surface roughness induces a decrease of the echo (real part or modulus) comparatively to the flat case. However, this decrease remains small owing to the small electromagnetic roughness of the surface at this typical frequency. Moreover, the dispersion around this mean value remains low. The general shape of the pdf resembles a Gaussian for the imaginary part and the phase. For the real part and the modulus, the shape differs only slightly from a Gaussian, and is assimilated to a Gaussian as well in first approximation.

The same qualitative observations can be made for the second echo $s_{2}$. In this case, the relative differences between the flat case and the mean value of the rough case are higher, owing to the larger electromagnetic roughness of the layer.

Second, in what follows the frequency behavior of the echoes $s_{1}$ and $s_{2}$ is investigated in the whole range of the two radar frequency bands under study, i.e. for $f \in[0.5 ; 3.0]$ $\mathrm{GHz}$, for which 1000 Monte-Carlo processes were used.

Fig. 3 presents the frequency behavior of the real part of the first two echoes $s_{1}$ and $s_{2}$. The flat case is plotted in green full line, the mean value of the rough case in red circled dashed line, the mean value plus or minus twice the standard deviation of the rough case in magenta circled dash-dot line,

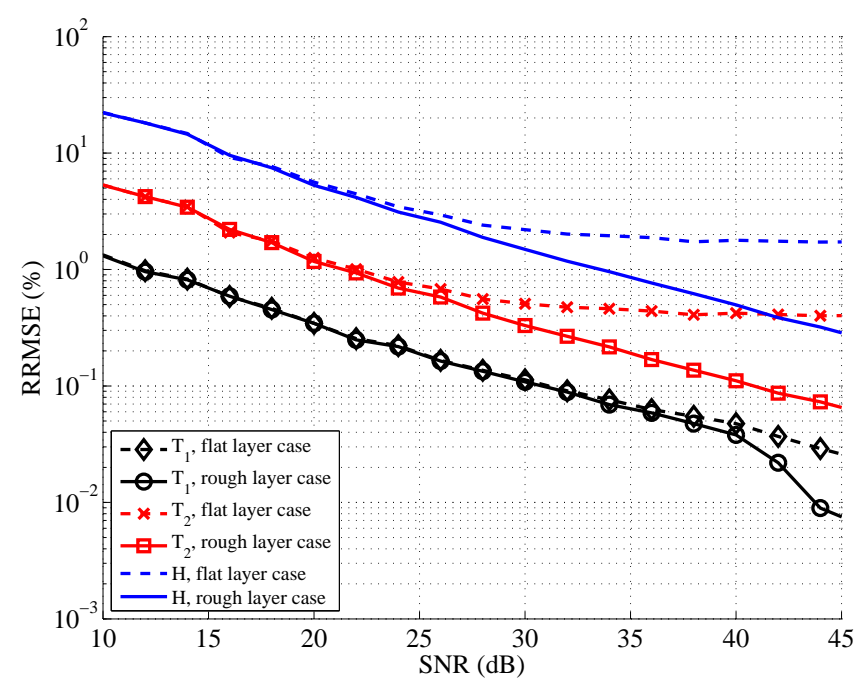

Fig. 4. RRMSE variations on the two estimated time delays $\hat{T}_{1}$ and $\hat{T}_{2}$, as well as on the layer thickness $\hat{H}$, vs. the SNR

and one realization of the rough case in blue dotted line with plus signs. The results highlight that as the radar frequency increases, the amplitudes of the backscattered echoes $s_{1}$ and $s_{2}$ decrease, because the layer (electromagnetic) roughness increases relatively to the wavelength. Moreover, for the lower frequencies $f \approx 1 \mathrm{GHz}$, it can be seen that the difference with the flat case is relatively weak and could be neglected. On the contrary, for the higher frequencies $f \approx 3 \mathrm{GHz}$, the relative difference with the flat case is significant and cannot be neglected any more, as it exceeds 10 percent for instance for $s_{2}$. Thus, significant differences appear in the backscattered echoes $s_{1}$ and $s_{2}$ between the rough and flat cases, in particular for the higher frequencies.

Then, let us have a look at the consequences of these differences in the electromagnetic backscattering on the thickness estimation by GPR, with the Maximum Likelihood Method (MLM).

\section{THICKNESS ESTIMATION BY GPR}

The process to determine the time delays of the first two echoes is explained in details in [3]. To perform time delay estimation (TDE), the MLM is used. An additive complex Gaussian white noise is considered to model the measurement uncertainties and the noise in the instruments. The radar pulse is a ricker pulse, defined as the second derivative of a Gaussian pulse. The data vector is made of 5 samples within the 2 $\mathrm{GHz}$ frequency bandwidth (see Fig. 3). The scenario under study is the same as described in the previous section. Thus, the data (i.e., the echo amplitudes $s_{1}$ and $s_{2}$ ) used to determine the time delays correspond to the realization plotted in blue dotted line with plus signs in Fig. 3.

Fig. 4 represents the relative root mean square error 
(RRMSE) variations on the two estimated time delays $\hat{T}_{1}$ and $\hat{T}_{2}$, as well as on the layer thickness $\hat{H}$, vs. the signal-to-noise ratio (SNR), for the frequency band $f \in[1.0 ; 3.0]$ GHz. First, for both flat and rough cases, it can be seen that the RRMSE decreases with increasing SNR. A difference between the flat and rough cases is observable in $\hat{T}_{1}$ for SNR higher than 40 $\mathrm{dB}$, in $\hat{T}_{2}$ for SNR higher than $25 \mathrm{~dB}$, and in $\hat{H}$ for SNR higher than $25 \mathrm{~dB}$.

As a consequence, taking the roughness of the surfaces into account makes it possible to increase the performances of the algorithm for moderate to high SNR. Thus, in the context of high SNR, it is important to take the roughness into account in the data modeling to obtain very low RRMSE, and this modeling allows in this case an even better precision of the thickness estimation. On the other hand, for low SNR and/or for a first estimate of the pavement thickness, these results confirm that taking the surface roughness into account is not necessary: this phenomenon can be neglected in this other context, as usually done in many previous studies.

\section{CONCLUSION}

In conclusion, taking the surface roughness into account in the pavement thickness estimation by standard GPR of bandwidth of the order of $2 \mathrm{GHz}$ allows us to quantify the classical approximation which considers flat interfaces. Thus, for typical pavements encountered in practice, like here $B=[1.0 ; 3.0]$ $\mathrm{GHz}$ in Fig. 4, the difference between the rough and the flat cases in the backscattered echoes amplitudes is significant for the higher frequencies of the bandwidth. Then, the influence of this difference in the GPR estimation process is significant for moderate to high SNR (typically, $25 \mathrm{~dB}$ for the thickness). As a consequence, taking the roughness into account in the data model is of interest, and this modeling allows a better precision in the thickness estimation process.

\section{REFERENCES}

[1] X. Dérobert, C. Fauchard, P. Côte, E. Le Brusq, E. Guillanton, J.Y. Dauvignac, and C. Pichot, "Step-frequency radar applied on thin road layers," Journal of Applied Geophysics, vol. 47, no. 3, pp. 317-325, July 2001.

[2] J.S. Lee, C. Nguyen, and T. Scullion, "A novel, compact, low-cost, impulse ground-penetrating radar for nondestructive evaluation of pavements," IEEE Transactions on Instrumentation and Measurement, vol. 53, no. 6, pp. 1502-9, Dec. 2004.

[3] C. Le Bastard, V. Baltazart, Y. Wang, and J. Saillard, "Thin-pavement thickness estimation using GPR with high-resolution and superresolution methods," IEEE Transactions on Geoscience and Remote Sensing, vol. 45, no. 8, pp. 2511-19, Aug. 2007.
[4] N. Déchamps, N. de Beaucoudrey, C. Bourlier, and $\mathrm{S}$. Toutain, "Fast numerical method for electromagnetic scattering by rough layered interfaces: Propagationinside-layer expansion method," Journal of the Optical Society of America A, vol. 23, no. 2, pp. 359-69, Feb. 2006.

[5] “AFNOR standard NFP 98-137," 1992, French standard.

[6] U. Spagnolini, "Permittivity measurements of multilayered media with monostatic pulse radar," IEEE Transactions on Geoscience and Remote Sensing, vol. 35, no. 2, pp. 454-63, Mar. 1997.

[7] G.G. Gentili and U. Spagnolini, "Electromagnetic inversion in monostatic ground penetrating radar: TEM horn calibration and application," IEEE Transactions on Geoscience and Remote Sensing, vol. 38, no. 4, pp. 1936-46, July 2000.

[8] M. Adous, X. Dérobert, and P. Quéffélec, "EM characterization of bituminous concretes in a large frequency bandwidth: First results," in The 11th International Conference on Ground Prenetrating Radar, Ohio, USA, June 2006.

[9] C. Fauchard, Utilisation de Radars très hautes fréquences : Application à l'auscultation non destructive des chaussées, Ph.D. thesis, University of Nantes, France, 2001.

[10] E.S. Li and K. Sarabandi, "Low grazing incidence millimeter-wave scattering models and measurements for various road surfaces," IEEE Transactions on Antennas and Propagation, vol. 47, no. 5, pp. 851-61, May 1999.

[11] F. Koudogbo, P.F. Combes, and H.-J. Mametsa, "Numerical and experimental validations of IEM for bistatic scattering from natural and manmade rough surfaces," Progress In Electromagnetics Research, vol. 46, pp. 203-244, 2004.

[12] F. Liu, Modélisation et Expérimentation Radar Impulsionnel et á Sauts de Fréquence Pour lAuscultation de Milieux Stratifiés du Génie Civil, Ph.D. thesis, University of Nantes, France, Apr. 2007.

[13] R. Dusséaux and R. De Oliveira, "Effect of the illumination length on the statistical distribution of the field scattered from one-dimensional random rough surfaces: analytical formulae derived from the small perturbation method," Waves in Random and Complex Media, vol. 17, no. 3, pp. 305-20, Aug. 2007. 